\title{
COMPARISON OF THE HIGH ENERGY MODELS FOR NEUTRAL MESON PHOTOPRODUCTION AND THE RELATED HADRONIC PROCESSES
}

\author{
F.D.GAULT and A.D.MARTIN \\ Department of Phystcs, University of Durham, England
}

and

G.L.KANE

Department of Physics, University of Michigan, Ann Arbor, USA

Recelved 26 May 1971

\begin{abstract}
To critically compare the Michigan and Argonne models we perform sumultaneous fits to the high-energy data for $\pi^{0}$ and $\eta$ photoproduction together with that for the related hadronic vector meson production reactions, in particular that for $\pi^{+} n \rightarrow \omega p$. Thus all constraints on these models are considered simultaneously. Particular attention is given to the strength of absorptive cuts in both models, and to the role of the B-meson exchange in filling dips in the Argonne model. We find that the $\rho$-Pomeron cuts (1.e. cuts associated with large helicity flip at the $N \bar{N}$ vertex) are essential to introducing unnatural parity exchange in the Michigan model, and that the B-meson contribution is incapable of filling unwanted dips which appear in any model with nonsense wrong signature zeros as required by exchange degeneracy. The flip and non-flip $\rho$ and $\omega$ coupling strengths at the $\mathrm{NN}$ vertex are determined. Predictions are made for varıous polarization asymmetries for $\gamma p \rightarrow \pi^{\circ} p$ and $\gamma p \rightarrow \eta p$. The differential cross section for $K_{L}^{0} p \rightarrow K_{S}^{O} p$ is calculated and compared with the existing data.
\end{abstract}

\section{INTRODUCTION}

Using the Reggeized absorption model [1-3] we study the high energy data for the neutral photoproduction reactions

$$
\begin{aligned}
& \gamma \mathrm{p} \rightarrow \pi^{\circ} \mathrm{p}, \\
& \gamma \mathrm{n} \rightarrow \pi^{\circ} \mathrm{n}, \\
& \gamma \mathrm{p} \rightarrow \eta \mathrm{p},
\end{aligned}
$$


together with data for the production of transversely polarized vector mesons

$$
\begin{aligned}
& \pi^{\mathrm{o}} \mathrm{p} \rightarrow \omega_{\mathrm{tr}} \mathrm{p} \\
& \pi^{\mathrm{o}} \mathrm{p} \rightarrow \rho_{\mathrm{tr}}^{\mathrm{o}} \mathrm{p}
\end{aligned}
$$

The cross sections for the latter reactions are determined from the measured cross sections for the isospin related reactions initiated by charged pions

$$
\begin{aligned}
& \frac{\mathrm{d} \sigma}{\mathrm{d} t}\left(\pi^{\mathrm{o}} \mathrm{p} \rightarrow \omega_{\mathrm{tr}} \mathrm{p}\right)=\rho_{11}^{\mathrm{H}} \frac{\mathrm{d} \sigma}{\mathrm{d} t}\left(\pi^{+} \mathrm{n} \rightarrow \omega \mathrm{p}\right), \\
& \frac{\mathrm{d} \sigma}{\mathrm{d} t}\left(\pi^{\mathrm{o}} \mathrm{p} \rightarrow \rho_{\mathrm{tr}}^{\mathrm{O}} \mathrm{p}\right)=2 \rho_{11}^{\mathrm{H}} \frac{\mathrm{d} \sigma}{\mathrm{d} t}\left(\pi^{\mathrm{o}} \mathrm{p} \rightarrow \rho^{\circ} \mathrm{p}\right) \\
& =\rho_{11}^{\mathrm{H}} \frac{\mathrm{d} \sigma}{\mathrm{d} t}\left(\pi^{+} \mathrm{p} \rightarrow \rho^{+} \mathrm{p}\right)+\rho_{11}^{\mathrm{H}} \frac{\mathrm{d} \sigma}{\mathrm{d} t}\left(\pi^{-} \mathrm{p} \rightarrow \rho^{-} \mathrm{p}\right)-\rho_{11}^{\mathrm{H}} \frac{\mathrm{d} \sigma}{\mathrm{d} t}\left(\pi^{-} \mathrm{p} \rightarrow \rho^{\mathrm{o}} \mathrm{n}\right),
\end{aligned}
$$

where $\rho^{\mathrm{H}}$ is the density matrix of the vector meson in the helicity frame. The connection between the photon processes ( 1 and 2 ) and the purely hadronic processes is made using the vector dominance model; that is, we assume the photon to be a coherent mixture of vector mesons, transversely polarized in the helicity frame (see for example ref. [4]).

A remarkable feature of the differential cross sections of the four processes at high energy is the correlation [5] of the presence or absence of dips at $t \cong-0.5$ $(\mathrm{GeV} / c)^{2}$ with the dominance of $\omega$ or $\rho$ meson exchange. The cross sections for processes (1) and (4) (for which $\omega$ exchange is thought to dominate) have a dip, whereas processes (2) and (3) (for which $\rho$ exchange is expected to dominate) have structureless cross sections in this region of $t$.

The ratio $\mathrm{d} \sigma / \mathrm{d} t\left(\gamma \mathrm{n} \rightarrow \pi^{\circ} \mathrm{n}\right) / \mathrm{d} \sigma / \mathrm{d} t\left(\gamma \mathrm{p} \rightarrow \pi^{\circ} \mathrm{p}\right)$ measured at $4 \mathrm{GeV}[6]$ indicates that there is some interference between the isoscalar ( $\rho$-exchange) and isovector ( $\omega$-exchange) parts of the photon amplitude in $\pi^{\circ}$ photoproduction, and measurements of the asymmetry parameter for linearly polarized photons $[7,8]$ show that the process is dominated by natural parity $(\rho, \omega)$ exchange, even in the region of the dip in the differential cross section. For the process $\pi^{+} n_{-} \rightarrow \omega p$ the quantity corresponding to the asymmetry is $\rho_{1,-1}^{\mathrm{H}} / \rho_{11}^{\mathrm{H}}$, and here again natural parity exchange is seen to dominate, though to a lesser degree.

In order to obtain a satisfactory description of the data in terms of the Regge model, it is necessary to introduce cut contributions (see, for example, ref. [9] for a review of the early fits to $\gamma \mathrm{p} \rightarrow \pi^{\circ} \mathrm{p}$ ). However the two conventionally used absorptive cut prescriptions give physically different explanations of the data.

In the Michigan model [2,3] the structure in the cross sections results from the destructive interference between the pole and cut contributions to the amplitudes. 
A feature of this model is that the Regge pole term is assumed to be free of nonsense wrong signature zeros (NWSZ). The cut contribution is then calculated in the usual way and multiplied by a $\lambda$ factor $(\lambda>1)$ to allow approximately for possible inelastic diffractive effects. Depending on the value of the $\lambda$-factor, the pole-cut interference can be arranged to produce a dip at $t \cong-0.5(\mathrm{GeV} / c)^{2}$ in amplitudes with net helicity flip $n=1$ and smooth behavior there for $n=0$ and 2. If one accepts $[5,10]$ that the $\omega N \bar{N}$ vertex is predominantly non-flip (leading to $n=1$ dominant amplitudes) and that $\rho \mathrm{NN}$ is flip (giving dominant amplitudes with $n=0$ and $n=2$ ), then one expects the model to reproduce the structure of the data.

For the Argonne model [1] on the other hand, the structure of the cross sections is essentially due to the presence of NWSZs in the amplitudes, and since pole terms change sign at these zeros, the cut contributions are correspondingly weaker. The problem of course arises that $\rho$ NWSZ dips near $t \cong-0.5(\mathrm{GeV} / c)^{2}$ are predicted for the essentially structureless $\gamma \mathrm{p} \rightarrow \eta \mathrm{p}$ and $\pi^{+} n \rightarrow \omega \mathrm{p}$ cross sections and it has been suggested [11] that the $B$ meson plays the important role of filling the unwanted dips. We therefore investigate whether or not a satisfactory description of the data for processes (1-4) can be achieved using the Argonne model with $\rho, \omega$ and B exchange.

In the conventional applications of the Argonne model the absorptive cut contributions are not multiplied by $\lambda$ factors. We find that this model gives a poor fit to the data. However since the $\lambda$ factors are found to play such a crucial role in the Michigan model we investigate whether or not the introduction of reasonable $\lambda$ factors can significantly improve the description of the data based on the Argonne model. We will call this the mixed model.

We emphasize that simultaneously analysing the available high energy data for the set of reactions $(1-4)$ is a stringent test of the models as the parameters needed to specify the $\pi^{\circ}$ photoproduction amplitudes are sufficient also to specify the amplitudes for reactions (2-4).

Finally, we mention other related analyses. There have been several analyses [12] of $\pi^{\circ}$ photoproduction which have included parametric forms for Regge cuts in the $t$-channel amplitudes. In all of these fits the poles were assumed to have NWSZs. The general conclusion is that the data can be well fitted with this extra freedom and that the cut contributions are very large. Blackmon et al. [13] fitted $\pi^{\mathbf{o}}$ photoproduction data in the Argonne model and found that, in order to fit the differential cross section and asymmetry data, they required a $B$ contribution with a high lying trajectory $\alpha_{B}=0.4+0.4 t$. They did not consider the other related processes.

Colocci [14] using certain simplifying assumptions has studied $\pi^{\circ}$ and $\eta$ photoproduction to see whether NWSZs are present or not and concludes that they are not. He did not, however, consider the possible effect of B exchange. Moreover, we note that lis cut contributions differ fundamentally from those used in the Argonne and Michigan models. This difference plays a significant role in his fit to the data, especially in his asymmetry predictions. Recently Kane et al. [15] used the Michigan 
model to fit neutral photoproduction* together with charged photoproduction and the nucleon-nucleon charge exchange reactions. However they did not consider $\omega$ production or fully use the constraints due to the $\pi^{\circ}$ asymmetry and the $\gamma \mathrm{n} \rightarrow \pi^{\circ} \mathrm{n} /$ $\gamma \mathrm{p} \rightarrow \pi^{\circ} \mathrm{p}$ cross section ratio.

Since the simultaneous fit to reactions $(1-4)$ impose severe constraints on the $\rho$ and $\omega$ couplings reliable estimates of the flip to non-flip ratios should be obtained. The parameterization that we use is described in sect. 2 . The fits to the data using the various models are presented in sect. 3 together with a discussion of the resulting coupling strengths. In sect. 4 predictions are made for certain photoproduction polarizations, and the $\mathrm{K}_{\mathrm{L}}^{\mathrm{o}} \mathrm{p} \rightarrow \mathrm{K}_{\mathrm{S}}^{\mathrm{o}} \mathrm{p}$ differential cross section is estimated and compared with the recent SLAC data [16]. Some conclusions are summarized in sect. 5.

\section{FORMALISM}

\section{1. s-channel amplitudes and cross section formulae}

We take the four independent $s$-channel helicity amplitudes, $T_{\mu^{\prime} \mu}^{\lambda}$, describing the photoproduction process $\gamma_{\lambda}+\mathrm{N}_{\mu} \rightarrow \mathrm{O}^{-}+\mathrm{N}_{\mu^{\prime}}$ to be $T_{++}^{1}, T_{--}^{1}, T_{-+}^{1}, T_{+-}^{1}$. The amplitudes are normalized such that the unpolarized differential cross section is

$$
\frac{\mathrm{d} \sigma}{\mathrm{d} t}=\frac{1}{128 \pi s q^{2}} \sum_{\mu \mu^{\prime}}\left|T_{\mu^{\prime} \mu}^{1}\right|^{2} .
$$

The releva.'t observable polarization cross sections can be easily expressed in terms of these amplitudes [17]. The asymmetry $\Sigma$ for scattering with linearly polarized photons is

$$
\Sigma \equiv \frac{\sigma_{\perp}-\sigma_{\| \prime}}{\sigma_{\perp}+\sigma_{\| \prime}}=2 \operatorname{Re}\left(T_{++}^{1} T_{--}^{1 *-} T_{-+}^{1} T_{+-}^{1}\right) / \sum_{\mu \mu^{\prime}}\left|T_{\mu^{\prime} \mu}^{1}\right|^{2}
$$

where $\sigma_{\perp}\left(\sigma_{/ /}\right)$is the differential cross section for photons polarized perpendicular (parallel) to the production plane. If the left-right asymmetry parameter $A$ for the scattering of unpolarized photons by a target of polarization $\mathcal{P}$ is defined by

$$
\mathrm{d} \sigma / \mathrm{d} t \propto 1+A \mathcal{P} \cdot \mathrm{n}
$$

where $\mathbf{n}$ is the normal to the production plane (Basel convention), then

$$
A=2 \operatorname{Im}\left(T_{-+}^{1 *} T_{--}^{1}-T_{+-}^{1} * T_{++}^{1}\right) / \sum_{\mu, \mu^{\prime}}\left|T_{\mu^{\prime} \mu}^{1}\right|^{2}
$$

* In relating the $\gamma p \rightarrow \eta p$ to the $\gamma p \rightarrow \pi^{\circ} p$ amplitudes the factor $1 / \sqrt{3}$ arising from the $\omega, \phi$ mixing was omitted in ref. [15] and, further, the amplitudes for $\rho$ and $\omega$ exchange were taken to have opposite sign in $\gamma \mathrm{p} \rightarrow \eta \mathrm{p}$. 
To describe the vector-meson production process $\mathrm{O}^{-}+\mathrm{N}_{\mu^{\prime}} \rightarrow \mathrm{V}_{\lambda}+\mathrm{N}_{\mu}$ we require two additional independent amplitudes, $T_{++}^{\circ}$ and $T_{-+}^{\circ}$. However these amplitudes enter neither the formula for the transverse cross section,

$$
\left(1-\rho_{\mathrm{oO}}^{\mathrm{H}}\right) \frac{\mathrm{d} \sigma}{\mathrm{d} t}=\frac{1}{64 \pi s q^{2}} \sum_{\mu, \mu^{\prime}}\left|T_{\mu^{\prime} \mu}^{1}\right|^{2},
$$

nor the "asymmetry" $\rho_{1,-1}^{\mathrm{H}} / \rho_{11}^{\mathrm{H}}$, where $\rho^{\mathrm{H}}$ is the spin density matrix of the vector meson in the $s$-channel helicity frame. The asymmetry is so called because it is given by an expression identical in form to that of eq. (6).

\subsection{Reggeization}

For both $\pi^{\circ}$ and $\eta$ photoproduction $\omega, \rho$ and B Regge exchange are allowed, whereas for process (3) $\omega$-exchange is forbidden and for process (4) only $\omega$-exchange is allowed. To leading order in $s$ the pole contribution to an $s$-channel helicity amplitude [18] resultıng from the exchange of a Reggeon, $i$, in the $t$-channel can be written

$$
\left(T_{\mu^{\prime} \mu}^{1}\right)^{\mathrm{P}}=\left(\sqrt{t_{\mathrm{o}}-t}\right)^{n+x} \mathrm{e}^{\frac{1}{2} i \pi J}\left(s_{\mathrm{o}}^{l}\right)^{J} \frac{G_{\mu^{\prime} \mu}^{t} N\left(\alpha_{i}\right)}{\sqrt{2}\left(m_{i}^{2}-t\right)}\left(\frac{s}{s_{\mathrm{o}}^{l}} \mathrm{e}^{-\frac{1}{2} i \pi}\right)^{\alpha_{l}(t)},
$$

where the net helicity flip $n=\left|\lambda-\left(\mu-\mu^{\prime}\right)\right|, x=|\lambda|+\left|\mu-\mu^{\prime}\right|-n$, and $m_{l}$ and $J$ are the mass and spin of the lowest particle on the trajectory. The $1 / \sqrt{2}$ is the product of normalisation factors 2 and $1 / 2 \sqrt{2}$ associated with the baryon and meson vertices respectively*. $t_{\mathrm{o}}$ is the value of $t$ at $\theta=0$, the forward direction $\left(t_{\mathrm{o}} \cong 0\right)$, and the factor $\left(s_{\mathrm{o}}^{i}\right)^{J}$ ensures that residue extrapolates to the particle coupling at $t=m_{l}^{2}$. The factor $N\left(\alpha_{i}\right)$ depends on the choosing mechanism of the trajectory at sensenonsense points. In the Michigan model $N\left(\alpha_{i}\right)=1$, and in the Argonne model $N\left(\alpha_{i}\right)$ $=\alpha_{i}$ for spin one exchange. For $\pi^{\circ}$ photoproduction the various coupling factors, $G_{\mu \prime \mu}^{l}$, are presented in table 1 , and are taken to be independent of $t$ in the fits to the data. The transverse vector-meson production amplitudes corresponding to the allowed Regge exchanges are also obtained from table 1 by omitting the photon vector-meson couplings $g_{\gamma} \mathrm{V}$. The amplitudes for $\gamma \mathrm{p} \rightarrow \eta \mathrm{p}$ may be related to those for $\gamma \mathrm{p} \rightarrow \pi^{\mathrm{o}} \mathrm{p}$ using SU(3) symmetry. Treating the photon as a $U$-spin scalar we find [21]

$$
\frac{\sqrt{3}}{A} T(\eta)=\frac{g_{\gamma \rho}}{g_{\gamma \omega}}\left[T_{\rho}\left(\pi^{\circ}\right)+T_{\mathrm{B}}\left(\pi^{\mathrm{o}}\right)\right]+\frac{g_{\gamma \omega}}{g_{\gamma \rho}} T_{\omega}\left(\pi^{\mathrm{o}}\right)
$$

where the factor $\sqrt{3}$ arises from the $\omega-\phi$ mixıng angle and $A=1.23$ arises from $\eta$-X mixing as given by the quadratic mass formula. Given that $g_{\gamma \rho} \cong 3 g_{\gamma \omega}$ we see that,

* See, for example, ref. [19] noting the correspondence $G \mathrm{~V}=\left(M g_{1}+g_{2}\right)$ and $G_{\mathrm{T}}=-M g_{1}$. 
Table 1

The couplings $G_{\mu^{\prime} \mu}^{l}$ for Reggeon $i$ exchange in $\gamma \mathrm{p} \rightarrow \pi^{\circ} \mathrm{p}$

\begin{tabular}{|c|c|c|c|c|c|}
\hline \multirow{2}{*}{$T_{\mu^{\prime} \mu}^{1}$} & \multirow[t]{2}{*}{$n$} & \multirow[t]{2}{*}{$x$} & \multicolumn{3}{|c|}{$G_{\mu^{\prime} \mu}^{l}$} \\
\hline & & & $\omega$-exchange & $\rho$-exchange & B-exchange \\
\hline$T_{++}^{1}$ & 1 & 0 & $g_{\rho \omega \pi} g_{\gamma \rho} G_{\mathrm{V}}^{\omega}$ & $g_{\rho \omega \pi} g_{\gamma \omega} G_{V}^{\rho}$ & 0 \\
\hline$T_{.-}^{1}$ & 1 & 0 & $g_{\rho \omega \pi} g_{\gamma \rho} G_{V}^{\omega}$ & $g_{\rho \omega \pi} g_{\gamma \omega} G \rho_{V}$ & 0 \\
\hline$T_{-+}^{1}$ & 0 & 2 & $-g_{\rho \omega \pi} g_{\gamma \rho} G_{\mathbf{T}}^{\omega} / 2 M$ & $-g_{\rho \omega \pi} g_{\gamma \omega} G_{T}^{\rho} / 2 M$ & $-g_{\omega \mathrm{B} \pi} g_{\gamma \omega} G_{\mathrm{T}}^{\mathrm{B}} / 2 M$ \\
\hline$T_{+-}^{1}$ & 2 & 0 & $+g_{\rho \omega \pi} g_{\gamma \rho} G_{\mathrm{T}}^{\omega} / 2 M$ & $+g_{\rho \omega \pi} g_{\gamma \omega} G_{T}^{P} / 2 M$ & $-g_{\omega \mathrm{B} \pi} g_{\gamma \omega} G_{\mathrm{T}}^{\mathrm{B}} / 2 M$ \\
\hline
\end{tabular}

The notation of ref. [15] is used. The coupling of the Reggeon at the N $\bar{N}$ vertex is calculated using $\bar{u}\left(\gamma_{\mu} G_{\mathrm{V}}+\sigma_{\mu \nu}\left(q_{\nu}-q_{\nu}^{\prime}\right) G_{\mathrm{T}} / 2 M\right) u$, where $M$ is the nucleon mass. The decay width for $\omega \rightarrow \pi^{\circ} \gamma$ gives $g_{\omega \pi \gamma}^{2} / 4 \pi=0.038$ [20]. The meson couplings are then determined from the vector dominance relation $g_{\omega \pi \gamma}=g_{\rho \omega \pi} g_{\gamma \rho}$ and $g_{\gamma \rho}=3 g_{\gamma \omega}=0.06$. (Units $\hbar=c=\mathrm{GeV}=1$.)

In going from $\pi^{\circ}$ to the $\eta$ photoproduction amplitudes, the $\rho$, B contributions are enzanced by about a factor of nine relative to that of the $\omega$-exchange contribution.

The pomeron exchange cut contribution associated with a given Regge exchange is calculated by evaluating the convolution integral analytically in the manner outlined in ref. [2]. We assume that the pomeron does not flip helicity and that it has a slope $\alpha_{\mathrm{p}}^{\prime}=0.5\left(\mathrm{GeV} / \mathrm{c}^{-2}\right.$. The details of the cut calculation are given in the appen$\operatorname{dix} \mathrm{A}$. The cut is multiplied by a constraned parameter $\lambda$ to allow approximately for possible inelastic diffractive effects. We allow these factors to be different for the contributions from the different Regge exchanges and, moreover, we distinguish between that associated with helicity flip $\left(\lambda_{f}\right)$ and that associated with non-flip $\left(\lambda_{\text {nf }}\right)$ at the $\mathrm{N} \overline{\mathrm{N}}$ vertex.

It is instructive to consider the origin of the factor $\left(\sqrt{t_{0}-t}\right)^{(n+x)}$ in the pole amplitude, especially as it requires all the pole contributions to vanish in the forward direction. The factor $\left(\sqrt{t_{\mathrm{o}}-t}\right)^{n}$ is required by angular momentum conservation. As each Regge exchange in the $t$-channel has a definite parity, parity conservation requires that $T_{-+}^{1}$ (net $s$-channel helicity flip $\left.n=0\right)$ be equal in magnitude to $T_{+-}^{1}(n=2)$. Since the latter vanishes as $\left(t_{0}-t\right)$ so must the former. The inclusion of absorptive cuts preserves the equality of the $n=1$ amplitudes while making the $n=0$ and $n=2$ amplitudes unequal. It is on this last point that Colocci [14] differs in that he has unequal cut contributions for $n=1$ amplitudes and equal cuts for $n=0$ and $n=2$ amplitudes.

The $\lambda$-factors are found to play a significant role in the description of the data, particularly in the Michigan model. This can be foreseen from fig. 1 on which we show typical Regge pole and cut contributions to the three relevant amplitudes. For the purposes of illustration we plot the modulus squared of the amplitude and of the 

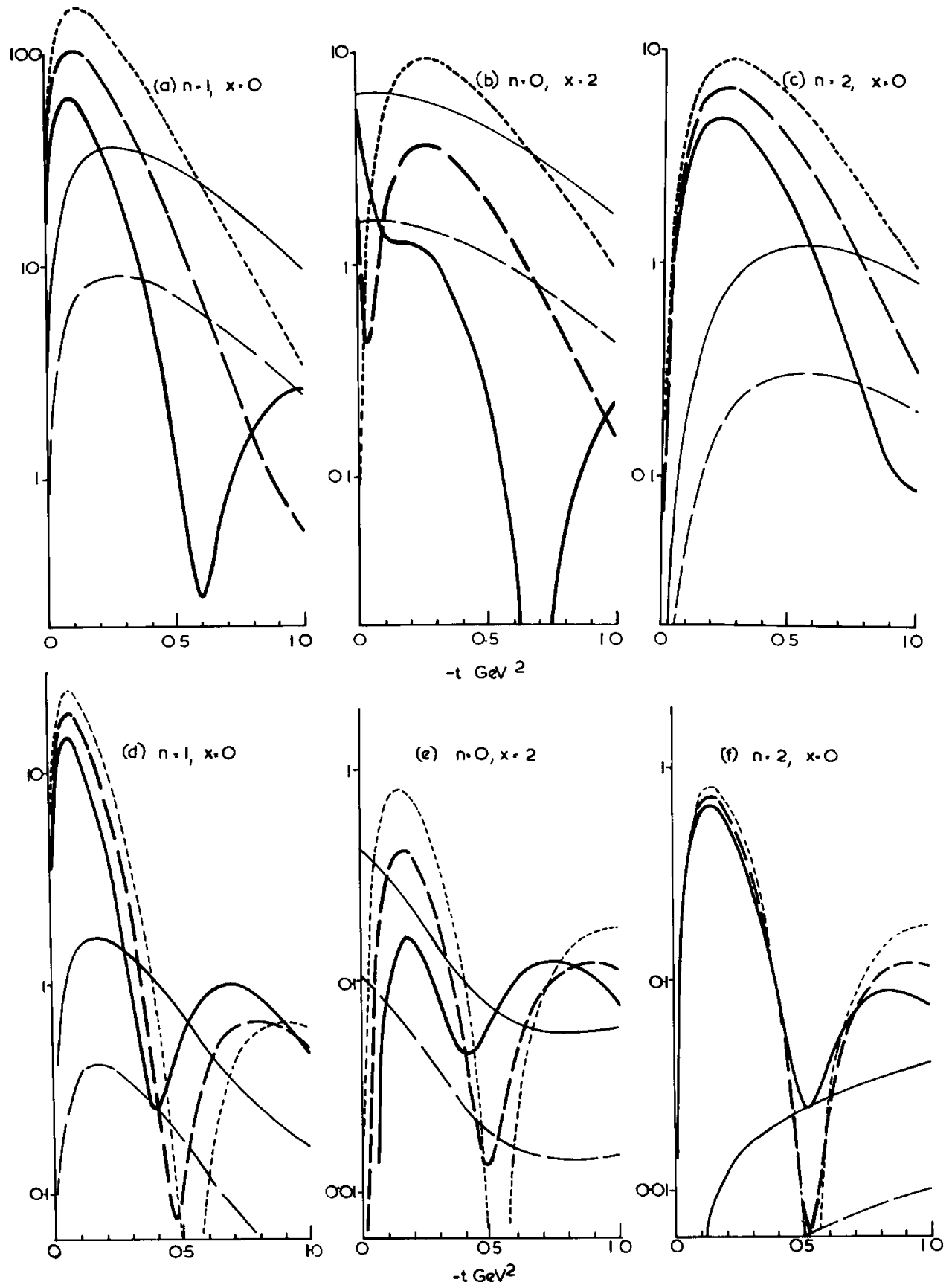

Fig. 1. For text see next page. 
Fig. 1. The amplitude is decomposed into its pole and cut contributions $T=T^{\mathrm{P}}+\lambda T^{\mathrm{c}}$. Here we plot the modulus squared of $T, T^{\mathrm{P}}$ and $\lambda T^{\mathrm{c}}$ for $\lambda=1$ and $\lambda=2$ for the three $(n, x)$ amplitudes for the $\omega$-exchange contribution to $\gamma \mathrm{p} \rightarrow \pi^{\circ} \mathrm{p}$ at $8 \mathrm{GeV} / c$ assuming that $G_{\mathrm{V}}=G_{\mathrm{T}}$. Figs. (a), (b), (c) correspond to the Michigan model and (d), (e), (f) are for a model with NWSZ. The pole contribution is shown by a dotted line. The dashed and contunuous lines correspond to $\lambda=1$ and $\lambda=2$ respectively. In each case, the fainter line corresponds to the cut and the darker line to the total contribution. The cut contribution is calculated from eq. (A.3) assuming $\alpha_{\mathbf{P}}^{\prime}=0.5$

$$
\mathrm{GeV}^{-2}, \sigma_{\mathrm{T}}=25 \mathrm{mb}, \rho=0 \text { and } A=8 \mathrm{GeV}^{-2} \text {. }
$$

pole and cut terms associated with $\omega$-exchange (w1th $G_{\mathrm{V}}=G_{\mathrm{T}}$ ) for $\lambda=1$ and $\lambda=2$. With equal flip and non-flip couplings the pole contributions to the $(n=1, x=0)$ amplitudes are larger in the relevant $t$ region than those to the $(0,2)$ and $(2,0)$ amplitudes by a factor $2 M / \sqrt{-t}$. This relative enhancement is reflected in the total ampltudes with the exception of the $(n=0, x=2)$ amplitude. The form of this amplitude is very sensitive to the value of $\lambda$ primarily because the cut, unlike the pole, contribution does not vanish at $t=t_{\mathrm{o}}$.

The effect of the $s_{o}$ parameters is less obvious than that of the $\lambda$-factors. However the variation of $s_{\mathrm{o}}$ is found to change the relative magnitude and $t$-dependence of the pole and cut contributions to the amplitude. In general, the smaller the value of $s_{\mathrm{o}}$, the stronger the cut contribution relative to the pole.

\section{ANALYSIS OF THE DATA AND THE COMPARISON OF THE MODELS}

The high energy data for reactions $(1-4)$ were fitted in the region* $|t|<1.1$ $(\mathrm{GeV} / \mathrm{c})^{2}$ using the Reggeized absorption models discussed in sect. 2. The parameterization was that of eq. (9). The couplings $G_{\mathrm{T}}^{l}$ and $G_{\mathrm{V}}^{l}$, associated with s-channel helicity flip and non-flip at the $N \bar{N}$ vertex respectively, were assumed to be independent of $t$ and were taken to be free parameters. However the parameter $s_{\mathrm{o}}^{i}$ associated with a Regge exchange can be interpreted as allowing some overall $t$-dependence of the residues. Each of these parameters was allowed to vary in the range $0.5<s_{\mathrm{o}}<2$. Linear trajectories, $\alpha(t)=\alpha_{\mathrm{o}}^{i}+\alpha_{1}^{i} t$, were used with $\alpha_{1}^{i}$ variable and $\alpha_{\mathrm{o}}^{i}$ constrained so that the lowest mass particle lay on the trajectory. The slopes of the $\rho$ and $\omega$ were confined to the interval $0.8<\alpha_{1}<1.0(\mathrm{GeV} / \mathrm{c})^{-2}$ and the B trajectory, which was used only in the Argonne and Mixed models, was fixed such that it passed through the $\pi$ meson [22] as well as the B, giving $\alpha_{B}=-0.014+$ $0.68 t$. The remaining parameters were the cut strength factors, $\lambda_{\mathrm{nf}}$ and $\lambda_{\mathrm{f}}$, associated with each Regge exchange. Depending upon the model, the $\lambda$-factors were fixed or bounded as shown in table 2 .

The Reggeon couplings at the meson vertex were fixed at the values given in table 1. An exception was that due to B-exchange and in this case the product $g_{\mathrm{B} \pi \omega} G_{\mathrm{T}}^{\mathrm{B}}$ was taken as the parameter. Since a crucial question [11] is whether B-

\footnotetext{
* The extreme forward data points in the Prumakoff region were omitted for the photoprocesses.
} 
Table 2

Range of variation of the $\lambda$-factors in the fits to the data

\begin{tabular}{ll}
\hline Model & Range \\
\hline Michigan & $1<\lambda_{\mathrm{nf}}<3,1<\lambda_{\mathrm{f}}<2$ \\
Argonne & $\lambda$ 's fixed at 1 \\
mixed & $1<\lambda<2$ \\
\hline
\end{tabular}

exchange is able to fill in the unwanted dips in absorption models with NWSZ we must allow this parameter to be at least as large as is physically reasonable. Various ways of estimating the upper bound are discussed in appendix $B$ and we concluded that the maximum possible value was about 400 . Therefore in order to be sure of resolving the role of B-exchange in the NWSZ absorption models we allowed $g_{\mathrm{B} \omega \pi} G_{\mathrm{T}}^{\mathrm{B}}$ to vary up to 800 in fits to the data.

The parameter values corresponding to the best fits to the data are listed in table 3 and the actual fits, to a selection of data, are shown in figs. 2-8. In these fits we took $\left(g_{\gamma \rho} / g_{\gamma \omega}\right)^{2}=9$ as required by SU(3) and 'ideal' $\omega-\phi$ mixing. However we repeated the analysis using the value, $\left(g_{\gamma \rho} / g_{\gamma \omega}\right)^{2}=7.1$, obtained by the Orsay storagering measurements [23]. This increase in the value of $g_{\gamma \omega}$ was found to make no essential change to the fits or to the parameter values. As expected it leads to a slight improvement in the fit to the $\gamma \mathrm{p} \rightarrow \eta \mathrm{p}$ cross section.

The Michigan model is seen to give a better overall fit than that of the Argonne model. We emphasize that in the latter model the inclusion of B-exchange with a large coupling is unable to remove the dips in $\pi^{+} n \rightarrow \omega p$ and $\eta$ photoproduction and moveover that the dip in $\pi^{\circ}$ photoproduction is still too deep. The Argonne description is improved if strong cuts are allowed. In fact we see that this mixed model gives a good fit to $\pi^{\circ}$ photoproduction, but even so the problems of unwanted structure near $t=-0.5$ persist in $\pi^{+} \mathrm{n} \rightarrow \omega \mathrm{p}$ and $\eta$ photoproduction.

Before we compare the various fits to the data in more detail let us consider the predictions for the $\rho$ and $\omega$ couplings at the $N \bar{N}$ vertex. We see that, independent of the models, the data require a predominantly s-helıcity flip $\rho N \bar{N}$ coupling and comparable flip $\left(G_{\mathrm{T}}\right)$ to non-flip $\left(G_{\mathrm{V}}\right) \omega \mathrm{N} \overline{\mathrm{N}}$ couplings in the region $t \lesssim 0$. These flip to non-flip ratios may be compared with the values $\left(G_{\mathrm{T}} / G_{\mathrm{V}}\right)_{\rho}=3.7$ and $\left(G_{\mathrm{T}} / G_{\mathrm{V}}\right)_{\omega}$ $=-0.14$ which are predicted from nucleon form factors, assuming that $\rho$ and $\omega$ are respectively the dominant isovector and isoscalar contributions. However to make this comparison we have to extrapolate the form factor values, first, from the photon $(J=1, t=0)$ to the vector meson mass $\left(J=1, t=m_{\mathrm{V}}^{2}\right)$, and then along the Regge trajectory to the scattering region $(t \leqslant 0)$.

In order to compare our ratios with other determinations [24] in the scattering region, $t \lesssim 0$, we note that the connection to the $t$-channel flip to non-flip ratio in terms of the conventional $\pi \mathrm{N} \rightarrow \pi \mathrm{N}$ amplitudes is 
Table 3

The values of the parameters obtained in the simultaneous fits to $\pi^{0}$ and $\eta$ photoproduction, $\pi^{+} \mathrm{n} \rightarrow \omega \mathrm{p}$ and $\pi^{\mathrm{o}} \mathrm{p} \rightarrow \rho^{\mathrm{o}} \mathrm{p}$.

\begin{tabular}{lccc}
\hline & & Model & \\
\cline { 2 - 4 } Parameter & Michigan & Argonne & mixed \\
\hline$G_{\mathrm{V}}^{\rho}$ & 3.2 & 4.7 & 5.8 \\
$G_{\mathrm{T}}^{\rho} / G_{\mathrm{V}}$ & 5.6 & 10.1 & 9.8 \\
$G_{\mathrm{V}}^{\omega}$ & 16.8 & 14.7 & 12.5 \\
$G_{\mathrm{T}}^{\omega} / G_{\mathrm{V}}^{\omega}$ & 1.3 & 1.0 & 1.5 \\
$g_{\mathrm{B} \omega \pi} G_{\mathrm{T}}^{\mathrm{B}}$ & & 800 & 800 \\
$\alpha_{1}^{\rho}$ & 0.89 & 0.81 & 0.81 \\
$\alpha_{1}^{\omega}$ & 0.99 & 0.89 & 0.81 \\
$s_{\mathrm{o}}^{\rho}$ & 1.00 & 0.57 & 0.50 \\
$s_{\mathrm{O}}^{\mathrm{B}}$ & 0.77 & 1.89 & 1.94 \\
$s_{\mathrm{o}}^{\mathrm{B}}$ & & 0.70 & 0.58 \\
$\lambda_{\text {nf }}^{\rho}$ & 2.43 & 1 (fixed) & 1.50 \\
$\lambda_{\mathrm{f}}^{\rho}$ & 2.00 & 1 (fixed) & 1.83 \\
$\lambda_{\mathrm{nf}}^{\omega}$ & 2.68 & 1 (fixed) & 2.0 \\
$\lambda_{\mathrm{f}}^{\mathrm{B}}$ & 1.52 & 1 (fixed) & 1.68 \\
$\lambda_{\mathrm{f}}^{\mathrm{B}}$ (fixed) & & 1 & 1 \\
$\chi^{2} /$ data pt. & 1.9 & 4.3 & 2.6 \\
\hline
\end{tabular}

$$
\left(\frac{\nu B}{A^{T}}\right)_{t=0}=1+\frac{G_{\mathrm{T}}}{G_{\mathrm{V}}}
$$

The values $\left(G_{\mathrm{T}} / G_{\mathrm{V}}\right)_{p}$ obtained in this analysis are in agreement with the values found from analyses of $\pi \mathrm{N}$ charge-exchange scattering. In fact by fitting the $\pi^{-} \mathrm{p}$ $\rightarrow \pi^{\circ} \mathrm{n}$ differential cross-section and polarization with the same form of parametrization we found $\left(G_{\mathrm{T}} / G_{\mathrm{V}}\right)_{\rho} \approx 8$ in the Argonne model and $\left(G_{\mathrm{T}} / G_{\mathrm{V}}\right)_{\rho} \approx 4$ in the Michigan model.

A comparison of the $\omega$ and $\rho$ couplings allows an estimate of the $D / F$ ratio for the $\mathrm{VB} \overline{\mathrm{B}}$ couplings within SU(3). We calculate the pole contributions (eq. (9)) to the amplitudes at $t=0$ using the parameters given in table 3 and then determine the $D / F$ ratio from the relation 


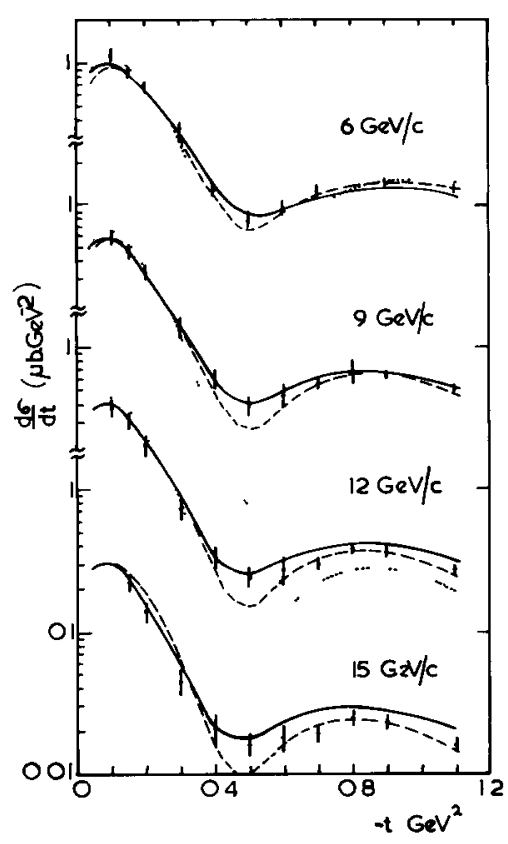

Fig. 2. The fit to the differential cross section for $\gamma \mathrm{p} \rightarrow \pi^{\circ} \mathrm{p}$. The data are from ref. [27]. The contunuous, dashed and dotted curves are for the Michigan, mixed and Argonne models respectively.

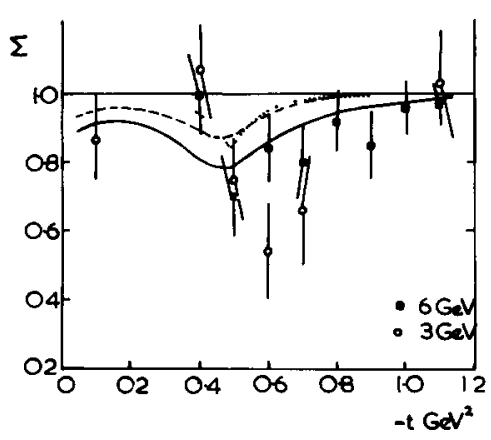

Fig. 3. The fit to the $3 \mathrm{GeV} / c$ [7] and 6 $\mathrm{GeV} / c[8]$ asymmetry data for scattering by linearly polarized photons for $\gamma \mathrm{p} \rightarrow \pi^{\circ} \mathrm{p}$. As the fits are essentially energy independent they are shown only at $6 \mathrm{GeV} / c$. The curves are as in fig. 2 .

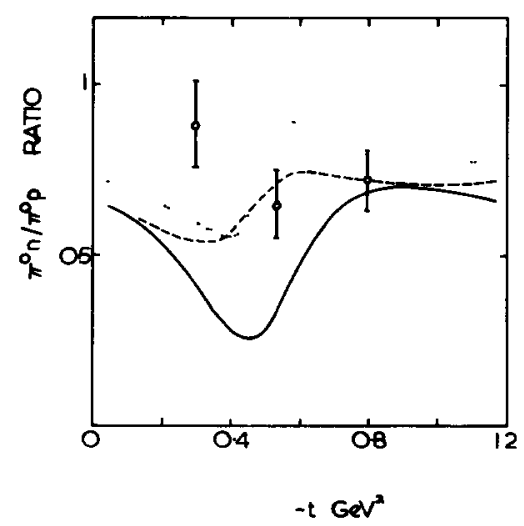

Fig. 4. The fit to the $4 \mathrm{GeV} / c$ data [6] for the ratio of the $\gamma \mathrm{n} \rightarrow \pi^{\circ} \mathrm{n}$ and $\gamma \mathrm{p} \rightarrow \pi^{\circ} \mathrm{p}$ differential cross sections. The curves are as in fig. 2 .

$$
\frac{T^{\omega}}{T^{\rho}} \equiv \frac{3 F-D}{F+D}
$$

The results are given in table 4 . The values for the $s$-channel non-flip couplings are in agreement with other determinations [24-26], while for the flip coupling ratio 

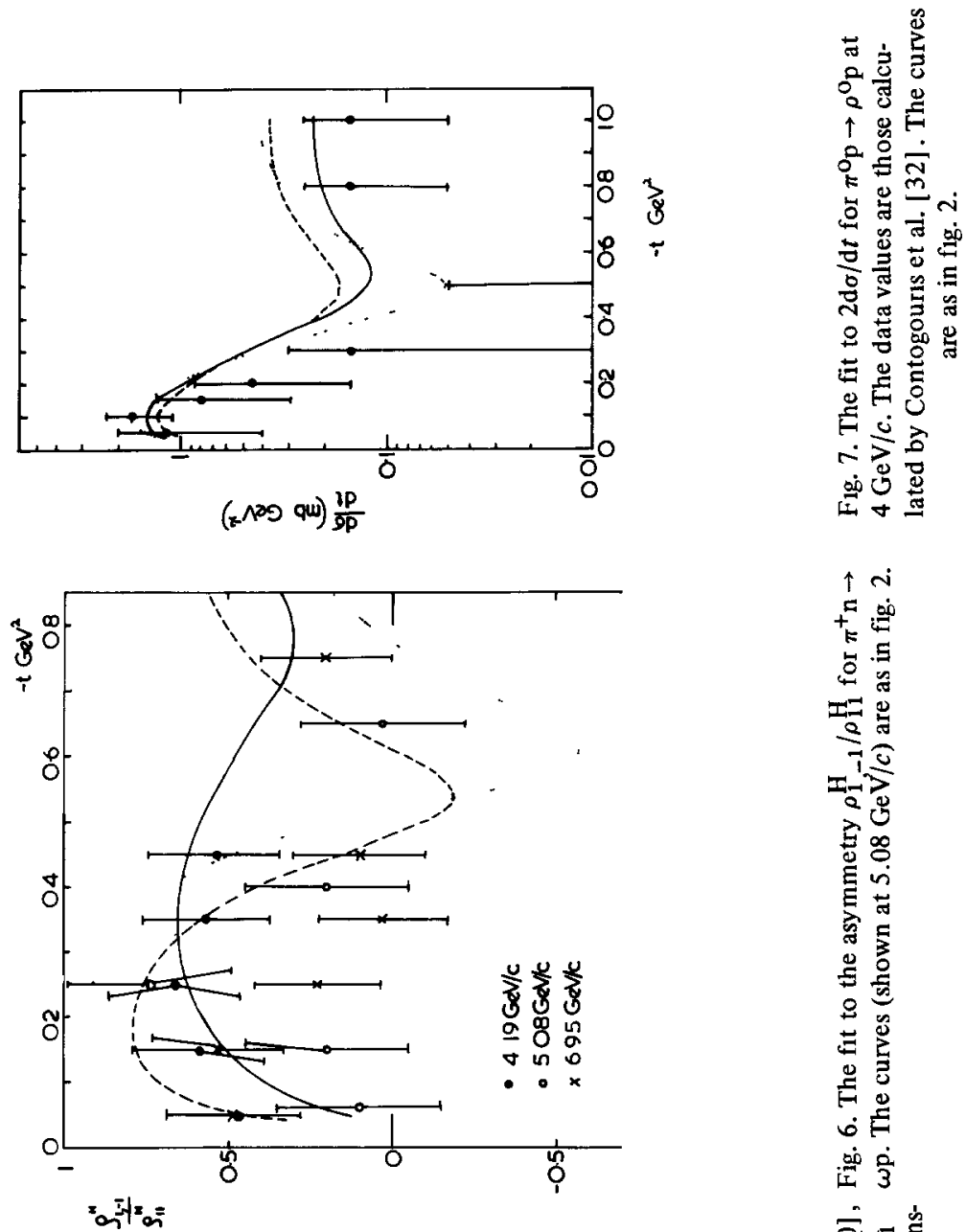

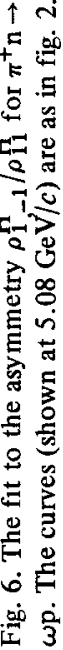

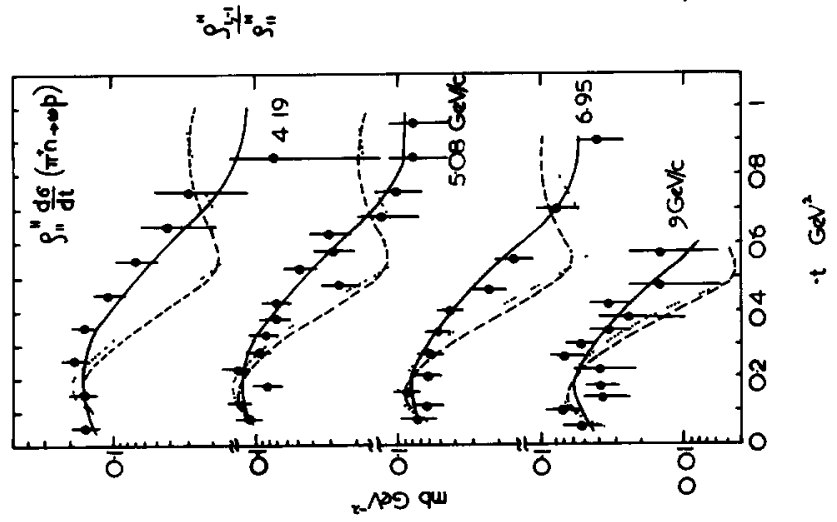

के=

कㄴㅇㅇ 은 in $\uparrow$ 要

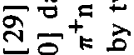
要曹 过高竞 号它 을 됴의

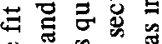

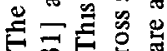
in ตู 


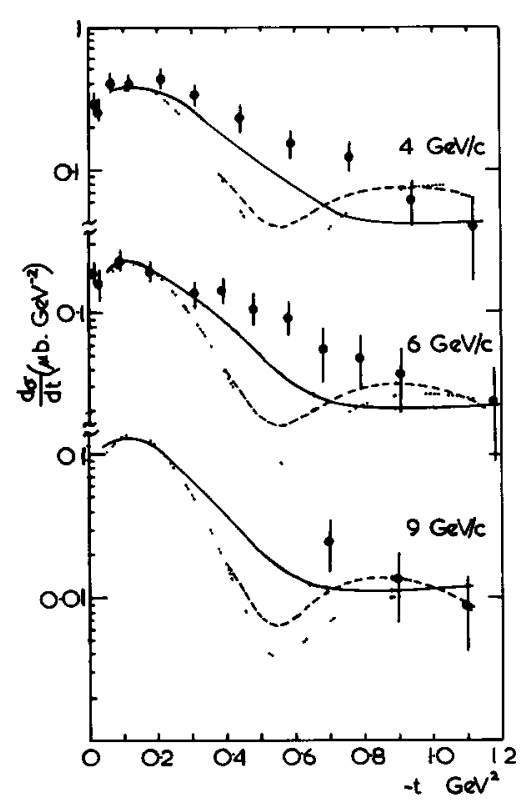

Fig. 8. The fit to the data for the $\gamma p \rightarrow \eta p$ differential cross section at 4 [34], 6 [34] and 9 [33] $\mathrm{GeV} / c$ The curves are as in fig. 2 .

Table 4

The ratios of the $\omega$ and $\rho$ pole amplitudes at $t=0$ and the corresponding $D / F$ ratios for the $\mathrm{VB} \overline{\mathrm{B}}$ couplings.

\begin{tabular}{lllll}
\hline \multirow{2}{*}{ Model } & \multicolumn{2}{c}{$s$-channel non-flip, $T_{++}$} & \multicolumn{2}{c}{$s$-channel flip, $T_{+-}$} \\
\cline { 2 - 5 } & $T \omega / T^{\rho}$ & $D / F$ & $T \omega / T^{\rho}$ & $D / F$ \\
\hline Michigan & 4.5 & -0.3 & 1.1 & 0.9 \\
Argonne & 5.8 & -0.4 & 0.6 & 1.5 \\
mixed & 4.2 & -0.2 & 0.6 & 1.4 \\
\hline
\end{tabular}

there does not seem to be any other reliable estımates*.

We conclude that the $\omega$ and $\rho$ couplings we have found in this analysis of $\pi^{\circ}$ photoproduction and related reactions are in general agreement with the expectations from other sources with the possible exception of $G_{\mathrm{T}}^{\omega}$. However these reactions probably afford the best means of determining the $\omega$ couplings. We note that the avalable data for these reactions do not fix the absolute sign of $\left(G_{\mathrm{T}} / G_{\mathrm{V}}\right)_{\rho}$ and $\left(G_{\mathrm{T}} / G_{\mathrm{V}}\right)_{\omega}$ but that they do determine their relative sign. Information on this over-

* In ref. [15] the parameter $\left(G_{\mathrm{T}} / G_{\mathrm{V}}\right)_{\omega}$ was upper bounded at 0.5 and so the ratio calculated from their parameters is not strictly comparable. 
all sign will come with measurements of the left-right asymmetry in $\pi^{\circ}$ photoproduction (cf. eq. (7)). We choose $\left(G_{\mathrm{T}} / G_{\mathrm{V}}\right)_{\rho}$ to be positive so as to be in agreement with sign predicted by the $\pi^{-} \mathrm{p} \rightarrow \pi^{\circ} \mathrm{n}$ polarization and the nucleon form factors.

We also notice from table 3 that, in both the NWSZ models $s_{\mathrm{o}}^{\rho}$ is small $(\approx 0.5)$ while $s_{\mathrm{o}}^{\omega}$ is large $(\approx 2)$. The small $s_{\mathrm{o}}^{\rho}$ might be anticipated from the data since it has the effect of enhancing the $\rho$ cut contribution relative to the pole term in the region of the NWSZ dip.

It is convenient to compare the descriptions of the data using the various models process by process.

\section{1. $\pi^{\mathrm{o}}$ Photoproduction}

The fits to the most recent $\gamma \mathrm{p} \rightarrow \pi^{\circ} \mathrm{p}$ differential cross section data, measured by Anderson et al. [27] are shown in fig. 2. These measurements show that the dip in the cross section data near $-t=0.5(\mathrm{GeV} / c)^{2}$ persists up to the highest energy observed. The critical parameter in the Michigan description is $\lambda_{n f}^{\omega}$, for which a value of $\lambda_{\mathrm{nf}}^{\omega}=2.7 \pm 0.1$ is essential to a reasonable $t$-dependence. The Argonne description has too deep a dip at $t=-0.5(\mathrm{GeV} / c)^{2}$, which is not surprising as the nonsense zeros of both the $\rho$ and $\omega$ contributions occur in the interval $(-0.4,-0.6)$. The low lying $B$ trajectory is unable to fill this excessive dip, a defect which is remedied in the mixed model by increasing $\lambda_{\mathrm{nf}}^{\omega}$ and consequently the corresponding cut contribution.

The asymmetry data $[7,8]$ for scattering by linearly polarized photons, fig. 3 , demonstrate the dominance of natural parity exchange. Some unnatural parity exchange, however, is indicated in the region of $t=-0.5(\mathrm{GeV} / c)^{2}$. In the models we have considered this can come from either the cut contributions associated with $\lambda_{f}$ [28] or from B-exchange, or both. This is the origin of the large $\lambda_{\mathrm{f}}^{\rho}$ in the Michigan model $\left(\lambda_{\mathrm{f}}^{\rho} \approx 2\right.$, as compared with $\lambda_{\mathrm{f}}^{\rho} \cong 1.2$ in the fit by Kane et al. [15]). From figs. $1(\mathrm{~b})$ and $1(\mathrm{c})$ we see that this maximuzes the difference between $T_{+-}^{1}$ and $T_{-+}^{1}$ and so gives the desired effect (see eq. (6)). In the Argonne model both the $\rho$ and $\omega$ contributions become small in the region of $t=-0.5(\mathrm{GeV} / c)^{2}$ and hence the unnatural parity B-exchange is able to give a dip in the asymmetry. In the mixed model, however, larger cut contributions increase the $\rho$ and $\omega$ contributions in the dip region and the effect of the $B$ is reduced. Although there is some unnatural parity gained from increasing the $\lambda_{\mathrm{f}}$ the effect is not as pronounced as in the Michigan model and the net result is that the mixed model gives less dip than the Argonne model for energies up to about $8 \mathrm{GeV} / c$.

In practice the situation could be more complex than that on which the absorption models are based and for $-t$ greater than 0.5 the pomeron may well have an $s$ channel helicity flip contribution. This would particularly affect the large $t$ asymmetry predictions since then unnatural parity exchange can be expected from the cut contributions associated with $\lambda_{n f}$. In this context we note that Colocci [14] does not use the absorptive prescription to calculate his cut contributions. As a consequence the deep dip in his asymmetry arises from the $\omega$ cut contribution associated with $\lambda_{\text {nf }}$. 
Although only a few measurements [6] of the ratio, $R$, of the $\gamma \mathrm{n} \rightarrow \pi^{\circ} \mathrm{n}$ and $\gamma \mathrm{p} \rightarrow \pi^{\circ} \mathrm{p}$ differential cross sections exist (fig. 4) they impose a severe constraint on the $\omega$ couplings relative to the $\rho$ couplings. In particular this data requires the $\omega$ exchange to have an $s$-helicity flip coupling sufficient for it to interfere with the predominantly flip $\rho$ and produce the observed values of $R<1$. We emphasize that improved data for this ratio would be very useful and would help to distinguish between the models.

\section{2. $\omega$-meson production}

Since the process $\pi^{+} n \rightarrow \omega p$ is expected to be dominated by $\rho$-exchange the data is of particular import to Regge theory. The two outstanding features of the data are, furst, the absence of a dip in the differential cross section near $t=-0.5$ and, second, that the $\rho_{\text {oo }}$ density matrix element of the $\omega$ is large. Here, of course, the main concern is the correlation of the transverse $\omega$ production data with the photoproduction data and not with the unexpected abundance of zero helicity $\omega$ mesons*. In order to do this the density matrix elements observed at 4.19 and $5.08 \mathrm{GeV} / c$ $[29,30]$ were rotated from the Gottfried-Jackson frame to the helicity frame, and compared with the measured values at $6.95 \mathrm{GeV} / c$ [31]. We found no evidence for any energy dependence of either the magnitude or the $t$-dependence of $\rho_{11}^{\mathrm{H}}$. Thus to obtain the $\pi^{+} \mathrm{n} \rightarrow \omega \mathrm{p}$ transverse cross section, $2 \rho_{11}^{\mathrm{H}} \mathrm{d} \sigma / \mathrm{d} t$, for those $t$-values corresponding to $\mathrm{d} \sigma / \mathrm{d} t$ measurements, a smooth curve was interpolated through the values of $\rho_{11}^{\mathrm{H}}$. The results are shown in fig. 5. The cross section is structu reless in the region of $t=-0.5(\mathrm{GeV} / c)^{2}$, however as there are few values of $\rho_{11}^{\mathrm{H}}$ beyond $|t|>0.4(\mathrm{GeV} / c)^{2}$ some structure in the transverse cross section cannot be ruled out. In the fits to this data the dominant contribution arises from $\rho$-exchange amplitudes with helicity flip $n \neq 1$ and the Michigan model well describes the cross section. The Argonne description on the other hand has a dip near $t=-0.6(\mathrm{GeV} / c)^{2}$ which neither B-exchange nor cut contribution is able to fill. Again allowing the $\lambda$ factors to vary improves the Argonne fit somewhat, but the structure due to the NWSZ remains.

The data for the asymmetry $\rho_{1,-1}^{\mathrm{H}} / \rho_{11}^{\mathrm{H}}$ are shown in fig. 6. This data, for which $\rho$-exchange dominates, should be compared with the photoproduction asymmetry, fig. 3, for which $\omega$ is the dominant exchange. The larger unnatural parity exchange in $\pi^{+} n \rightarrow \omega p$, as compared with that in $\gamma p \rightarrow \pi^{\circ} p$, is explained for our models if we note that it arises essentially from the absorptive cuts associated with the $\rho \mathrm{N} \bar{N}$ flip amplitudes. In the Michigan model the fit to the data depends critically upon the large value of $\lambda_{\rho}^{\mathrm{f}}$. If $\lambda_{\rho}^{\mathrm{f}}$ is constrained to 1.2 we find $\rho_{1,-1}^{\mathrm{H}} / \rho_{11}^{\mathrm{H}} \approx 0.9$ essentially independent of $t$. Characteristic of the Argonne model is the deep dip at $-0.6(\mathrm{GeV} / c)^{2}$ although in fig. 6 it is enhanced by the large value of the B coupling. Here again improved data could help to distinguish between the models.

* In our models these would be attributed to B-exchange, however the relevant coupling is different from the one involved here and so no prediction can be made (see appendix $B$ for a comment on this). 
We have investigated the effects of the $\rho-\omega$ electromagnetic mixıng on the $\pi^{+} n$ $\rightarrow \omega$ p data. Due to the large $\pi$-exchange peak in $\pi^{+} n \rightarrow \rho^{\circ}$ p data this mixing can have an appreciable effect on the $\pi^{+} n \rightarrow \omega$ p measurements at small $t$, particularly on the density matrix element $\rho_{\mathrm{OO}}^{\mathrm{GJ}}$. This effect could be of consequence to zero-helicity $\omega$ production, but it does not appreciably affect the transverse cross section, although it may increase $\rho_{1,-1}^{\mathrm{H}} / \rho_{11}^{\mathrm{H}}$ by as much as 0.15 for small $t$-values.

\section{3. $\rho$-meson production}

As compared to $\omega$ production, it is more complicated to obtain $\rho$-production data which can be connected with $\pi^{\circ}$ photoproduction. In principle a value for the $\pi^{\mathrm{o}} \mathrm{p} \rightarrow \rho^{\mathrm{o}} \mathrm{p}$ cross section is obtained by calculating

$$
1 / 2\left[\mathrm{~d} \sigma / \mathrm{d} t\left(\pi^{+} \mathrm{p} \rightarrow \rho^{+} \mathrm{p}\right)+\mathrm{d} \sigma / \mathrm{d} t\left(\pi^{-} \mathrm{p} \rightarrow \rho^{-} \mathrm{p}\right)-\mathrm{d} \sigma / \mathrm{d} t\left(\pi^{-} \mathrm{p} \rightarrow \rho^{\circ} \mathrm{n}\right)\right]
$$

from the existing data. This combination eliminates $\pi$-exchange and is expected to be dominated by $\omega$-exchange. However large cancellations occur in forming the combination, particularly for $-t<0.3$, and the final result is particularly susceptible to any errors in normalization of the data. As an example we show in fig. 7 the cross section calculated by Contogouris et al. [32] from the $4 \mathrm{GeV} / c$ data. Since in our models the $\rho$-meson density matrix element $\rho_{11}^{\mathrm{H}}=\frac{1}{2}$ these data can be regarded as the transverse cross section. From the figure we see that all models are able to reproduce the required $t$-dependence and, due to the large errors inherent in these data, they do not play a significant role in the fit.

\section{4. $\eta$ photoproduction}

We have related $\eta$ photoproduction to the above three reactions using SU(3) symmetry for the meson couplings together with the $\omega-\phi$ mixing as predicted by $\mathrm{SU}(6)$ or the quark model (cf. eq. (10)). From fig. 8 we see that the $\gamma p \rightarrow \eta p$ cross section data $[33,34]$ is well fitted with these assumptions for small $t$. However the models predict a cross section too low by a factor of 2 or 3 near $t=-0.5(\mathrm{GeV} / \mathrm{c})^{2}$ which could be attributed to symmetry breaking effects.

From the couplings given in table 3 we see that $\rho$-exchange dominates for this process, though the $\omega$ contribution is by no means negligible. Because $\rho$ dominates we expect the Michigan model to predict a structureless cross section near $t=-0.5$ $(\mathrm{GeV} / \mathrm{c})^{2}$, however the Argonne and mixed models still show the effects of the NWSZ even with B-exchange present.

\section{PREDICTIONS}

The measurement of polarisation observables for the photoprocesses will be able to shed further light on the $t$-dependence of amplitudes and so help to distinguish between the models [35]. 


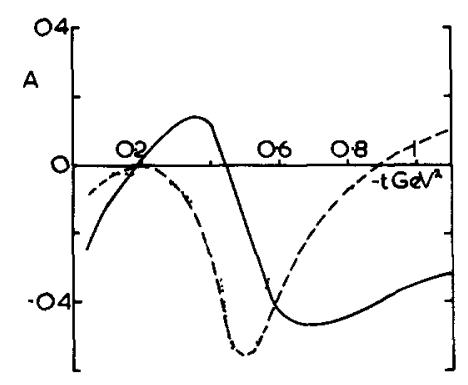

F1g. 9. The predictions for the $\gamma \mathrm{p} \rightarrow \pi^{\circ} \mathrm{p}$ left-right asymmetry for scatterıng from a polarized target at $6 \mathrm{GeV} / c$. The contınuous, dashed and dotted curves are for the Michigan, mixed and Argonne models respectively.

In fig. 9 we show the predictions for the left-right asymmetry parameter, $A$, for $\gamma \mathrm{p} \rightarrow \pi^{\mathrm{o}} \mathrm{p}$ for scattering from a polarized target. Another observable is the recoll proton polarization in an experiment where the target and photon beam are unpolarized. In general this is different from the asymmetry $A$. However, since $T_{++}^{1}=$ $T_{-}^{1}$ in the absorption models*, they predict that the recoil polarization (in the direction of the normal to the reaction plane) and the asymmetry are equal. This equality depends on the pomeron conserving s-channel helicity and so may well be violated for $-t \gtrsim 0.5$.

We recall that from the fit to the available data we were unable to determine the overall sign of the couplings. We see from eq. (7) that this sign can be obtained from measurements of this asymmetry.

In fig. 10 we show the predictions for the asymmetries $\Sigma$ and $A$ for $\gamma \mathrm{p} \rightarrow \eta p$. As $\rho$-exchange is important for this process the asymmetry $\Sigma$ for scattering with linearly polarized photons should be similar to $\rho_{1,-1}^{\mathrm{H}} / \rho_{11}^{\mathrm{H}}$ for $\pi^{+} \mathrm{n} \rightarrow \omega \mathrm{p}$. The remarks of subsect. 3.2 therefore apply.

The process $\mathrm{K}_{\mathrm{L}}^{\mathrm{o}} \mathrm{p} \rightarrow \mathrm{K}_{\mathrm{S}}^{\mathrm{o}} \mathrm{p}$ should proceed through $\omega$ - and $\rho$-exchange. Here again $\phi$ exchange is expected to be negligible because of the small $\phi p \bar{p}$ coupling. We can therefore estimate the $K_{L}^{o} p \rightarrow K_{S}^{o} p$ cross section by taking the baryon couplings from table 3 and relating the meson couplings to $p \pi \pi$ through SU(3). Then to leading order in $s$ the pole contributions to the $s$-channel helicity amplitudes are

$$
T_{++}^{i}=i \frac{N\left(\alpha_{i}\right) g_{\rho \pi \pi}}{m_{i}^{2}-t} s_{\mathrm{o}}^{l} G_{\mathrm{V}}^{i}\left(\frac{s}{s_{\mathrm{o}}^{l}} \mathrm{e}^{-\frac{1}{2} l \pi}\right)^{\alpha_{i}(t)}
$$

* Note that for charged photoproduction $T_{++}^{1} \neq T_{-.}^{1}$ if $A_{1}$ exchange is included. $A_{1}$ exchange is forbidden for $\gamma p \rightarrow \pi^{\circ} p$. 

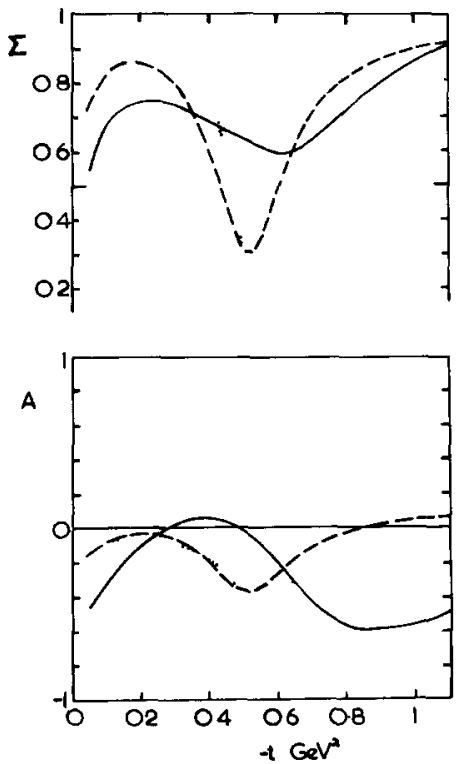

Fig. 10. The predictions for the $\gamma p \rightarrow \eta p$ asymmetries at $6 \mathrm{GeV} / c$, (a) for scattering with linearly polarzed photons (b) for scattering from a polarized target. The curves are as in fig. 9.
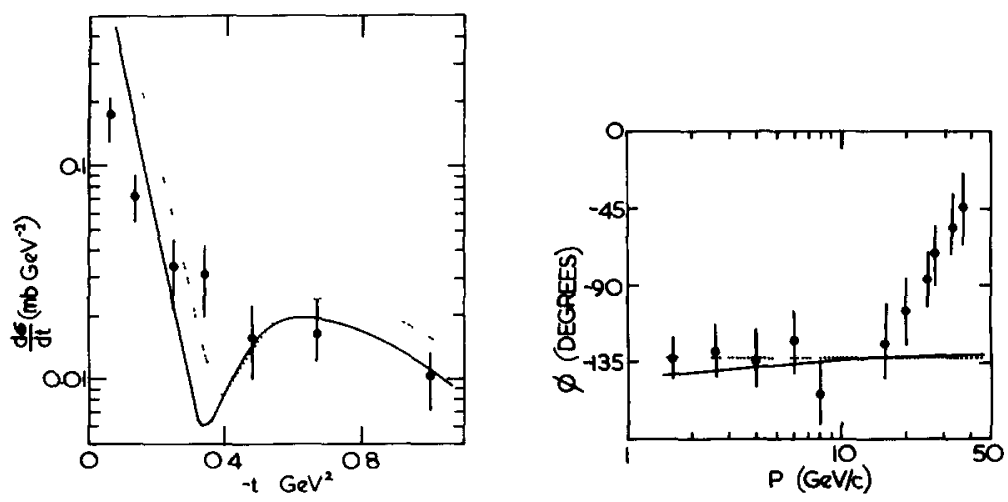

Fig. 11(a). The prediction made at $5.5 \mathrm{GeV} / c$, to the data for the $\mathrm{K}_{\mathrm{L}}^{\mathrm{o}} \mathrm{p} \rightarrow \mathrm{K}_{\mathrm{S}}^{0} \mathrm{p}$ differential cross section averaged over 4-8 GeV/c [16]. The coupling and trajectory parameters are taken from table 3. (b) The prediction to the data for the phase of the forward $\mathrm{K}_{\mathrm{L}}^{\mathrm{O}} \mathrm{p} \rightarrow \mathrm{K}_{\mathrm{S}}^{\mathrm{O}} \mathrm{p}$ amplitude. The data below $10 \mathrm{GeV} / c$ are from ref. [16], and data above $10 \mathrm{GeV} / c$ are from ref. [36]. The continuous and dotted curves correspond to the Michigan and Argonne predictions respectively. 


$$
T_{+-}^{l}=-i \sqrt{-t} \frac{N\left(\alpha_{l}\right) g_{\rho \pi \pi}}{m_{i}^{2}-t} \frac{s_{\mathrm{o}}^{l} G_{\mathrm{T}}^{l}}{2 M}\left(\frac{s}{s_{\mathrm{o}}^{i}} \mathrm{e}^{-\frac{1}{2} i \pi}\right)^{\alpha_{i}(t)},
$$

where we have used the notation of eq. (9). The combination $T=T^{\omega}-T^{\rho}$ gives the $\mathrm{K}_{\mathrm{L}}^{\mathrm{o}} \mathrm{p} \rightarrow \mathrm{K}_{\mathrm{S}}^{\mathrm{o}} \mathrm{p}$ amplitudes.

Here we test the compatability of the models with the data [16] for the $K_{L}^{o} p \rightarrow$ $\mathrm{K}_{\mathrm{S}}^{\circ} \mathrm{p}$ cross section and with the observed values $[16,36]$ of the phase $\phi=\tan ^{-1}$ $\left(\operatorname{Im} T_{++} / \operatorname{Re} T_{++}\right)_{t=0}$. The curves are obtained using the $\rho$ and $\omega$ parameters of table 3 together with $g_{\rho \pi \pi}^{2} / 4 \pi=2.5$. For the Michigan model the $\lambda$-factors were fixed at 1.6, whereas for the Argonne prediction we have, as usual, $\lambda=1$. Both models are in good agreement with the cross section data for $|t|>0.2$, but are found to over-estimate the observed forward peak. The dip in the Michigan prediction of the cross section is associated with the $\omega$ pole-cut interference in the nonflıp $(n=x=0)$ amplitude, whereas that in the Argonne description is due to the NWSZ's. The phase of the forward amplitude is well reproduced below $10 \mathrm{GeV} / c$ but, as has been noted [26,37], the models disagree with the preliminary measurements made at Serpukhov.

It can be argued that the values of the $s_{\mathrm{o}}^{i}$ in eq. (12) should not be taken from table 3, and so we fitted to the available $K_{L}^{o} p \rightarrow K_{S}^{o} p$ data with $s_{o}^{\omega}$ and $s_{\text {e }}^{\rho}$ as free parameters, but keeping the other quantities unchanged. For the value $s_{0}^{\omega}=0.37$ a good overall Michigan fit to the cross section is obtained. In fact this two-parameter fit gives a similar description to the five-parameter SCRAM fit of Johnson et al. [26] (note their fixed $E_{\mathrm{o}}=0.17$ ), in particular the forward peak is well described and the non-flip dip is turned into a shoulder. On the other hand the variation of the $s_{\mathrm{o}}^{i}$ parameters does not markedly improve the Argonne description. However here again the forward peak can be reduced by decreasing $s_{\mathrm{o}}^{\omega}$.

\section{CONCLUSIONS}

The main results obtained in the Michigan, Argonne and mixed model fits to the data of reactions (1-4) may be summarized as follows:

(1) In all models the $\rho N \bar{N}$ coupling is predominantly $s$-helicity flip, consistent with that needed to fit $\pi \mathrm{N}$ charge exchange data.

(ii) In all models the $\omega N \bar{N}$ coupling has comparable s-helicity flip and non-flip components $\left(G_{\mathrm{T}} \approx G_{\mathrm{V}}\right)$.

(iii) The $\rho$ and $\omega$ parameters predict $D / F \approx-0.3$ for the $s$-channel non-flip $\operatorname{VB} \bar{B}$ coupling, and an $s$-channel flip $D / F$ ratio in the range 1 to 1.5 (see table 4 ).

(iv) The Argonne model gives a poor overall fit even with the inclusion of B-exchange with a large coupling.

(v) In both the Michigan and the mixed models large cut contributions are required (that is some $\lambda$-factors in the range of 2 to 3 ).

(v1) Given these large cuts the Michigan model gives a satisfactory overall fit, 
while the mixed description, although an improvement on the Argonne model, still has unwanted structure near $t=-0.5$ in the $\rho$-dominated processes.

(vii) The $\lambda_{\mathrm{f}}^{\rho}$ factor plays a crucial role in introducing unnatural parity exchange in the Michigan model.

(vii) Further data on the various polarization observables for the photoprocesses and on the $\gamma \mathrm{n} \rightarrow \pi^{\circ} \mathrm{n} / \gamma \mathrm{p} \rightarrow \pi^{\circ} \mathrm{p}$ cross-section ratio would lead to a better determınation of the amplitudes and so distinguish between the various high energy models.

(ix) The fact that reasonable overall fits can be obtained lends support to the vector dominance assumptions as a prescription for relating photonic and hadronic amplitudes. The only exception is the $\mathrm{SU}(3)$ related $\gamma \mathrm{p} \rightarrow \eta \mathrm{p}$ cross section. This fit could be improved by allowing symmetry breaking effects.

While there are ways of improving both the Michigan and Argonne fits (such as allowing the pomeron to flip helicities) the object of this paper has been to compare the two models subject to the same set of physically reasonable constraints. The fit favours the Michigan model as compared to the Argonne and mixed models. The unwanted dips in the Argonne model for the processes dominated by $\rho$-exchange (e.g. $\pi^{+} n \rightarrow \omega p$ ) remain a problem; neither the inclusion of B-exchange nor of large absorptive cuts are able to provide a reasonable description of this data in a NWSZ model.

We thank Dr. R.J.N.Phıllips for the hospitality extended to us at the Rutherford Laboratory where part of this work was carried out. We are indebted to the CERN DD division for supplying a copy of the library program MINUIT. We also thank Mr. D.Harrison and Mrs. B.Chadwick for assistance in the collection of the data. The support of the S.R.C. 1s acknowledged.

\section{APPENDIX A}

The absorption correction $T^{\mathrm{c}}$ to the Regge pole amplitude $T^{\mathrm{P}}$. is calculated by analy tically evaluating the convolution integrals in the manner described in ref. [2] . The elastic scattering amplitude is expressed in the form

$$
T_{\lambda^{\prime} \mu^{\prime \prime} \lambda \mu}^{\mathrm{el}}=-4 q^{2} \sigma_{\mathrm{T}}(\mathrm{i}+\rho) \mathrm{e}^{A^{\prime} t / 2} \delta_{\lambda^{\prime} \lambda} \delta_{\mu^{\prime} \mu},
$$

where $\sigma_{\mathrm{T}}$ is the total cross section, $\rho$ the ratio of the real to imaginary parts of the forward scattering amplitude, $A^{\prime}=A-i \pi \alpha_{\mathrm{P}}^{\prime}, \alpha_{\mathrm{P}}^{\prime}$ is the slope of the pomeron and $A$ the slope of the elastic scatterıng diffraction peak, $A \approx 8 \mathrm{GeV}^{-2}$. Using

$$
\frac{1}{m^{2}-t}=\frac{1}{m^{2}}\left(1+t^{2} / 2 m^{4}\right) \mathrm{e}^{t / m^{2}}+O\left(t^{3}\right)
$$

we may approxımate the Regge pole amplitude, $T^{\mathrm{P}}$ of eq. (9), by 


$$
\begin{aligned}
T_{\mu^{\prime} \mu}^{\mathrm{P}} & =(\sqrt{-t})^{n+x} \mathrm{e}^{\frac{1}{2} i \pi J} \frac{\left(s_{\mathrm{o}}\right)^{J} G_{\mu^{\prime} \mu^{\prime} N(\alpha)}}{\sqrt{2} m^{2}}\left(\frac{s}{s_{\mathrm{o}}} \mathrm{e}^{-\frac{1}{2} i \pi}\right)^{\alpha_{\mathrm{O}}}\left(1+\frac{t}{2 m^{4}}\right) \mathrm{e}^{\frac{1}{2} B t} \\
& =(-t)^{\frac{1}{2} n} \sum_{k}\left(c_{\mu^{\prime} \mu}\right)_{k}\left(\frac{1}{2} t\right)^{k} \mathrm{e}^{\frac{1}{2} B t} \\
& =(-t)^{\frac{1}{2} n} \sum_{k}\left(c_{\mu^{\prime} \mu}\right)_{k} \frac{\partial^{k}}{\partial B^{k}}\left(\mathrm{e}^{\frac{1}{2} B t}\right)
\end{aligned}
$$

where $\alpha=\alpha_{0}+\alpha_{1} t$

$$
B=2\left[1 / m^{2}+\alpha_{1}\left(\ln \left(s / s_{\mathrm{o}}\right)-\frac{1}{2} i \pi\right)\right],
$$

and $c_{k}$ are known coefficients, independent of $t$. Using eqs. (A.1) and (A.2) the absorption correction can be shown [2] to be

$$
T_{\mu^{\prime} \mu}^{\mathrm{c}}=-(-t)^{\frac{1}{2} n} \frac{\sigma_{\mathrm{T}}(1-i \rho)}{4 \pi A^{\prime}} \sum_{k}\left(c_{\mu^{\prime} \mu}\right)_{k} \frac{\partial^{k}}{\partial B^{k}}\left[\left(\frac{A^{\prime}}{A^{\prime}+B}\right)^{n+1} \exp \left(\frac{\frac{1}{2} A^{\prime} B t}{A^{\prime}+B}\right)\right] .
$$

Although for simplicity we have set $t_{\mathrm{o}}=0$ it is straightforward to modify the result for $t_{\mathrm{o}} \neq 0$.

\section{APPENDIX B}

One of the objects of our analysis is to investigate the role of B-exchange in a NWSZ absorption model. In the fits to the data the strength of the B-exchange contribution is parametrized as the product of the couplings $g_{\mathrm{B} \omega \pi}$ and $G_{\mathrm{T}}^{\mathrm{B}}$. Here we estimate an upper bound for this parameter.

One source of information is the width of the decay $B \rightarrow \pi \omega$. A complication is that the decay of such an axial vector meson occurs through a mixture of two different orbital angular momentum states (s and d) [38] and so two couplings are required to specify the $B \pi \omega$ vertex

$$
m=i \epsilon_{\nu}\left(k_{\mathrm{B}}\right)\left[\frac{g_{1}}{m_{\mathrm{B}}} Q_{\nu} Q_{\mu}+g_{2} m_{\mathrm{B}} g_{\mu \nu}\right] \epsilon_{\mu}\left(k_{\omega}\right)
$$

where $Q=\frac{1}{2}\left(k_{\omega}-k_{\pi}\right)$ and where the amplitude $\mathcal{M}$ is normalized as in ref. [39]. The decay width can be expressed in terms of these two dimensionless couplings, $g_{i}$, as follows 


$$
\Gamma(\mathrm{B} \rightarrow \pi \omega)=\frac{p_{\mathrm{f}}}{96 \pi m_{\mathrm{B}}^{2} m_{\omega}^{2}}\left\{\left[g_{1} p_{\mathrm{f}}^{2}-g_{2}\left(m_{\mathrm{B}}^{2}+m_{\omega}^{2}-m_{\pi}^{2}\right)\right]^{2}+8 m_{\omega}^{2} m_{\mathrm{B}}^{2} g_{2}^{2}\right\}
$$

where $p_{\mathrm{f}}$ is the three-momentum of the decay products in the rest frame of the Bmeson. The coupling $g_{\mathrm{B} \omega \pi}$ of table 1 , required in the calculation of the B-exchange contribution to the transverse cross sections, turns out to be simply $g_{1}$ when extrapolated to the particle pole. Our problem is therefore to estimate a limit for $g_{1}$. Using the observed decay width $\Gamma(B \rightarrow \pi \omega)=130 \mathrm{MeV}$ we find from eq. (B.2) that $\left|g_{1}\right|<72$. However a value of the coupling near this upper bound is very unlikely, since such a.large value corresponds to an almost pure d-wave decay at an energy not much above the $\pi \omega$ threshold.

Further information on the coupling can be obtained from the data [40] for $\pi^{-} \mathrm{p} \rightarrow \mathrm{B}^{-} \mathrm{p}$ that exists at $5 \mathrm{GeV} / c$. Assuming that the $\omega$-exchange contribution to the s-channel amplitude $T_{++}^{\mathrm{o}}$ is dominant at $t=0$ we have

$$
\left.\frac{\mathrm{do}}{\mathrm{d} t}\right|_{t=0} \approx \frac{\left(\alpha_{\mathrm{o}}^{\omega} \chi\right)^{2}}{64 \pi q^{2} s}\left[\frac{2 s_{\mathrm{o}}^{\omega} G_{\mathrm{V}}^{\omega}}{m_{\omega}^{2}}\left(g_{2}-\frac{1}{4} g_{1}\right)\right]^{2}\left|i\left(\frac{s}{s_{\mathrm{o}}^{\omega}} \mathrm{e}^{-\frac{1}{2} l \pi}\right)^{\alpha_{\mathrm{o}}^{\omega}}\right|^{2},
$$

where $\alpha_{\mathrm{o}}^{\omega} \approx 0.5$ and $G_{\mathrm{V}}^{\omega}, s_{\mathrm{o}}^{\omega}$ are taken from table 3 . We have included a NWSZ factor and we let $\chi=0.7 ; \chi$ approximates the effect of absorption on the $T_{++}^{o}$ amplitude at $t=0$ in such a model. We expect small corrections to eq. (B.3) from the $\omega$-P cut contrıbution to $T_{+-}^{1}$, which does not vanish at $t=0$, and from $\mathrm{A}_{2}$ exchange. However to the accuracy we require it is reasonable to neglect these terms. We use the observed $\pi^{-} \mathrm{p} \rightarrow \mathrm{B}^{-} \mathrm{p}$ distribution and $\sigma \approx 50 \mu \mathrm{b}$ [41] to evaluate eq. (B.3) and we obtain $\left|g_{2}-\frac{1}{4} g_{1}\right| \approx 0.7$. Combining this result with the decay width constraint of eq. (B.2) gives $\left|g_{1}\right|<20$.

Other than the exchange degenerate arguments mentioned in the next paragraph we have no means of estimating $G_{\mathrm{T}}^{\mathrm{B}}$ but it is reasonable to suppose that $\left|G_{\mathrm{T}}^{\mathrm{B}}\right|<20$. Thus a physically reasonable upper bound for $g_{\mathrm{B} \omega \pi} G_{\mathrm{T}}^{\mathrm{B}}$ is about 400 . We note that if we come to the conclusion, from the fits to the data, that such a large B coupling accounts for the deficiencies of the Argonne model, it will then be hard to understand the energy dependence and magnitude of the observed ratio of $\gamma \mathrm{n} \rightarrow \pi^{-} \mathrm{p}$ and $\gamma \mathrm{p} \rightarrow$ $\pi^{+} n$.

For comparison we also calculate the value of the required $B$ coupling that is obtained from exchange degeneracy arguments. First by considering $\rho_{\mathrm{oo}} \mathrm{d} \sigma / \mathrm{d} t$ for the exotic reaction $\mathrm{K}^{+} \mathrm{n} \rightarrow \mathrm{K}^{* 0} \mathrm{p}$ we find that the $\pi$ and $\mathrm{B}$ exchanges are degenerate [22] . It then follows, assuming $\mathrm{SU}(3)$ and a vanishing $\mathrm{B} \phi \pi$ coupling, that the $\pi$-exchange in $\pi^{+} n \rightarrow \rho^{\circ} p$ and B-exchange in $\pi^{+} n \rightarrow \omega p$ have the same residue functions. Using the known values for $\pi$-exchange we calculate $g_{\mathrm{B} \omega \pi} G_{\mathrm{T}}^{\mathbf{B}} \approx 140$.

The consequences of a large value of $g_{1}$ are not confined to the transverse amplitudes $T_{\mu^{\prime} \mu}^{1}$. The B-exchange coupling for the $\pi^{+} \mathrm{n} \rightarrow \omega \mathrm{p}$ longitudinal amplitude $T_{+-}^{\mathrm{o}}$ is proportional to 


$$
\left(\frac{\sqrt{t} k_{t}}{2 m_{\mathrm{B}}} g_{1}-m_{\mathrm{B}} \frac{k_{\mathrm{o}}}{k_{t}} g_{2}\right) \frac{G_{\mathrm{T}}^{\mathrm{B}}}{2 M}
$$

where $k_{t}$ is the $t$-channel c.m. momentum and $k_{\mathrm{o}}^{2}=k_{t}^{2}+m_{\omega}^{2}$. Thus a large $g_{1}$ could lead to a large value of $\rho_{\mathrm{oo}} \mathrm{d} \sigma / \mathrm{d} t$ for $\pi^{+} \mathrm{n} \rightarrow \omega \mathrm{p}$.

\section{REFERENCES}

[1] R.C.Arnold, Phys. Rev. 153 (1967) 1523.

[2] F.Henyey, G.L.Kane, J.Pumplin and M.H.Ross, Phys. Rev. 182 (1969) 1579.

[3] M.Ross, F.S.Henyey and G.L.Kane, Nucl. Phys. B 23 (1970) 269.

[4] J.J.Sakura, Proc. of the IV Int. Symposium on electron and photon interactions at high energies (Liverpool, 1969) and references therein.

[5] H.Harari, Proc. of the IV Int. Symposium on electron and photon interactions at high energies (Liverpool, 1969).

[6] G.C.Bolon et al., reported by K.Lübelsmeyer, Proc. of the IV Int. Symposium on electron and photon interactions at high energies (Liverpool, 1969).

[7] D.Bellanger et al., Phys. Rev. Lett. 23 (1969) 540.

[8] R.Anderson et al., Phys. Rev. Lett. 26 (1971) 30.

[9] R.Diebold, Proc. of high energy phy sics conference (Boulder, 1969).

[10] R.Odorico, R.Garcia and C.A.Garcia-Canal, Phys. Lett. 32B (1970) 375.

[11] J.Tran Thanh Van, Nuovo Cim. Lett. 3 (1970) 678.

[12] J.Frфyland, Nucl. Phys. B11 (1969) 204;

A.P.Contogour1s, J.P.Lebrun and G.Von Bochman, Nucl. Phys. B13 (1969) 246;

A.Capella and J.Tran Thanh Van, Nuovo Cim. Lett. 1 (1969) 321;

M.Braunschweig, W.Braunschweig, D.Husmann, K.Lubelsmeyer and D.Schmitz, Nucl. Phys. B20 (1970) 191.

[13] M.L.Blackmon, G.Kramer and K.Schllung, Phys. Rev. 183 (1969) 1452.

[14] M.Colocc1, Nuovo Cim. Lett. 4 (1970) 53.

[15] G.L.Kane, F.Henyey, D.R.Richards, M.Ross and G.Willaamson, Phys. Rev. Lett. 25 (1970) 1519.

[16] A.Brody et al., Phys. Rev. Lett. 26 (1971) 1050.

[17] J.D.Jackson and C.Quigg, Nucl. Phys. B22 (1970) 301.

[18] G.Cohen-Tannoudji, Ph.Salın and A.Morel, Nuovo Cum. 55A (1968) 412.

[19] F.D.Gault and H.F.Jones, Nucl. Phys., B30 (1971) 68.

[20] A.Dar, T.L.Watts and V.F.Weisskopf, Phys. Lett. 30B (1969) 264.

[21] B.Gorczyca and M.Hayashi, Acta Phys. Polonica 36 (1969) 47.

[22] A.S.Goldhaber, G.C.Fox and C.Quigg, Phys. Lett. 30B (1969) 249;

G.V.Dass, Proc. of Daresbury study weekend 12-14 June, 1970: Vector meson production and omega-rho interference, A.Donnachie and E.Gabathuler eds., DNPL/R7/1970;

G.S.Abrams and U.Maor, Phys. Rev. Lett. 25 (1970) 621.

[23] J.E.Augustın et al., Phys. Lett. 28B (1969) 508.

[24] C.Mıchael, Proc. of Ruhestein meeting, ed. G.Hohler, Springer Tracts in Mod. Phys., vol. 55 (1970) 176.

[25] C.Michael and R.Odorico, Phys. Lett. 34B (1971) 422.

[26] W.B.Johnson et al., Phys. Rev. Lett. 26 (1971) 1053.

[27] R.L.Anderson et al., SLAC-PUB-770 (1970).

[28] F.Henyey, K.Kajantie and G.L.Kane, Phys. Rev. Lett. 21 (1968) 1782.

[29] G.S.Abrams et al., Phys. Rev. Lett. 23 (1969) 673. 
[30] N.Armenise et al., BBFO collaboration, private communication from J.Tran Thanh Van.

[31] J.A.J.Matthews et al., Phys. Rev. Lett. 26 (1971) 400.

[32] A.P.Contogouris, J.Tran Thanh Van and H.J.Lubatti, Phys. Rev. Lett. 19 (1967) 1352.

[33] R.Anderson et al., Phys. Rev. D1 (1970) 27.

[34] W.Braunschweig et al., Phys. Lett. 33B (1970) 236.

[35] E.L.Berger and G.C.Fox, Phys. Rev. Lett. 25 (1970) 1783.

[36] Dubna-Serpukhov collaboration, contribution Kiev conference (1970); J.V.Allaby, CERN Report 70-2989.

[37] V.Barger and R.J.N.Phillips, Phys. Lett. 33B (1970) 425.

[38] J.Ballam et al., Phys. Rev. D1 (1970) 94.

[39] J.D.Jackson and H.Pilkhun, Nuovo Cim. 33 (1964) 906.

[40] G.Ascoli, H.B.Crawley, D.W.Mortara and A.Shapiro, Phys. Rev. Lett. 20 (1968) 1411.

[41] D.W.Mortara, private communication. 